\title{
The photo-physical study of the interaction of riboflavin with Nicotine in bicontinuous microemulsion
}

\author{
Maurice O. Iwunze \\ Department of chemistry, Morgan State University, Baltimore, MD 21251, USA
}

\begin{abstract}
Steady-state fluorescence spectroscopy was used to study the interaction of riboflavin with nicotine in a bicontinuous microemulsion system made up of 42.11:13.70:21.34:22.85 \% w/w of water: oil: surfactant: cosurfactant. The surfactant used is cetyltrimethylammonium bromide (CTAB), the oil is tetradecane and the cosurfactant is 1-pentanol. It is observed that the interaction of riboflavin and nicotine in the prepared microemulsion lead to the quenching of riboflavin fluorescence. The bimolecular quenching rate constant, $\mathrm{k}_{\mathrm{q}}$, of riboflavin by nicotine was observed as $4.15 \times 10^{9} \mathrm{M}^{-1} \mathrm{~s}^{-1}$ with an efficiency of $56 \%$. The mechanism of the reaction is proposed to be diffusion limited in an activated electron transfer reaction in a solvent separated (outer-sphere) scheme. The electron transfer rate constant, $\mathrm{k}_{\mathrm{ET}}$, was calculated as $5.89 \times 10^{9} \mathrm{~s}^{-1}$ with an activation rate constant, $\mathrm{k}_{\mathrm{a}}$, of $9.52 \mathrm{x}$ $10^{9} \mathrm{~s}^{-1}$. The calculated solvent reorganization energy, $\lambda_{\mathrm{s}}$, of the reaction was $1.09 \mathrm{eV}$, the free energy of interaction, $\Delta \mathrm{G}^{\mathrm{o}}$, is $-2.9 \mathrm{eV}$ and the free energy of activation, $\Delta \mathrm{G}^{*}$, was calculated as $0.75 \mathrm{eV}$.
\end{abstract}

Keywords: Riboflavin, nicotine, fluorescence, Electron transfer, bicontinuous microemulsion, CTAB.

\section{Introduction}

Riboflavin (Vitamin $\mathrm{B}_{2}$ ) belongs to a family of flavins and is a cofactor in the activity of many flavorenzymes. It is a very versatile and important compound in chemistry, biology and medicine ${ }^{1-6}$. Articles reviewing the importance, utility and activities of this compound whose structure is shown in Fig. 1 have been given by Powers ${ }^{7}$ and Massey ${ }^{8}$.<smiles>Cc1cc2nc3c(=O)[nH]c(=O)nc-3n(CC(O)C(O)C(O)CO)c2cc1C</smiles>

Figure 1. Chemical Structure of riboflavin

In addition to its numerous biological activities, it is known to have the ability to generate singlet oxygen radical, ${ }^{1} \dot{\mathrm{O}}_{2},{ }^{9-12}$. Riboflavin is fluorescent and this feature has been used in photo-oxygenation of damaging antigens and other disrupting biomolecules in electron transport system 1, 10,13-15. On the other hand, nicotine is an organic alkaloid of formula $\mathrm{C}_{10} \mathrm{H}_{14} \mathrm{~N}_{2}$. It is a hygroscopic alkaline compound that is soluble in water.
It is found in the nightshade of family plants but it is predominant in the tobacco plant and its major concentration is in the leaves of this plant where it is observed that its concentration is about 0.3 to $0.5 \%{ }^{16-20}$. Its chemical structure of nicotine is shown in Fig. 2 and its chemistry, metabolism, kinetics and its use as biomarker has been delineated by Benowitz and his co-workers ${ }^{21}$. 
<smiles>CN1CCC[C@H]1c1cccnc1</smiles>

Figure 2. Chemical structure of Nicotine

The addictive nature of this alkaloid is well known ${ }^{22-26}$. The literature is replete of the notorious feature of nicotine. Among other negative features of this compound is its effect in increasing heart rate, increase in blood pressure and respiration for those indulged in the smoking cigarette which produces enormous amount of nicotine ${ }^{16,26-28}$. Also, nicotine has been recognized as an inhibitor in the essential electron transport system in biological processes. It is therefore desirable to degrade it before it has the chance to effect this deleterious inhibition. Riboflavin's ability to generate singlet oxygen can be used as a catalyst for degradation of nicotine via the photodynamic therapy (PDT) type of mechanism. This approach has been used by Janda and his coworkers ${ }^{10}$. In this work, we carry out the steady-state fluorescence quenching of riboflavin by nicotine to obtain the necessary physico-chemical data to enable a meaningful formulation of the mechanism for the degradation of this notoriously addictive compound.

\section{Experimental}

\section{Chemicals}

98.0\% pure nicotine and analytical reagent grade riboflavin were obtained from Sigma-Aldrich Chemicals. Cetyltrimethylammonium bromide (CTAB) of $99 \%$ purity and $99 \%$ pure tetradecane were obtained from Acros Organics. Analytical reagent grade quinine sulfate and 1-pentanol were obtained from Fisher Scientific. These chemicals were used as received.

\section{Instrument}

The fluorescence spectra were obtained from Perkin Elmer's luminescence spectrophotometer, model LS 50B. The Cary spectrophotometer, model $1 \mathrm{E}$, supplied by Varian Analytical Instrument Co. was used in obtaining the UV-Vis spectra. All electrochemical experiments were conducted using a computer-controlled electrochemical analyzer, model CS-1090 supplied by Cypress Systems. Leica Auto Abbe refractometer was used to obtain all the refractive indices used in this work, and the viscosity of the bicontinuous microemulsion was determined using the Brookfield viscometer model RDVD II+.

\section{Methodology}

\section{Bicontinuous Microemulsion Preparation}

Appropriate volume of water was added to a weighed amount of surfactant (CTAB). The mixture was gently stirred, and it was initially slurry. A calculated volume of oil (tetradecane) was then added and this produced a two-phase system. After that a cosurfactant (1-pentanol) was added drop-wise while the system is being stirred. After a given amount of cosurfactant was added, a clear isotopic solution was obtained. This system remained clear and homogeneous for a long time. This signifies its thermodynamic stability. Table 1 gives the respective weights, volume, volume fraction and percentage of the chemicals used to obtain the bicontinuous microemulsion used in this work.

Table 1. The Chemical Composition of the Components of Bicontinuous Microemulsion.

\begin{tabular}{|l|l|l|l|l|}
\hline Component \% & Weight, g & Volume, mL & Weight & Volume Fraction \\
\hline Water & 103.75 & 103.75 & 42.11 & 0.38 \\
\hline Surfactant (CTAB) & 52.57 & 52.84 & 21.34 & 0.20 \\
\hline Oil (tetradecane) & 33.75 & $44.00(\rho=0.767 \mathrm{~g} / \mathrm{mL})^{*}$ & 13.70 & 0.16 \\
\hline $\begin{array}{l}\text { Co-Surfactant } \\
(\mathbf{1}-\text { pentanol) }\end{array}$ & 56.30 & $69.16(\rho=0.814 \mathrm{~g} / \mathrm{mL}) *$ & 22.85 & 0.26 \\
\hline
\end{tabular}

*Densities of these solvents were taken from the manufacturer's stipulations.

Optical Measurements.

All the fluorescence spectra were obtained using a clear four-sided 1-cm quartz cuvette. The excitation wavelength was set at $401.00 \mathrm{~nm}$, and the emission was observed between 529 and $530 \mathrm{~nm}$. The excitation and emission slit widths were kept constant at 5 nanometers, respectively. On the other hand, the absorption measurements were made using a twoclear sided 1-cm quartz cuvette. The concentration of riboflavin was kept constant at $1.169 \times 10^{-4} \mathrm{M}$ while the concentration of nicotine was varied from $1.091 \mathrm{x}$ $10^{-3}$ to $1.910 \times 10^{-2} \mathrm{M}$. This is also the concentrations used in the fluorescence measurements experiments. The wavelength of maximum absorbance, $\lambda_{\max }$, of 
riboflavin in bicontinuous microemulsion was observed at $415 \mathrm{~nm}$. At this wavelength the molar absorptivity, $\varepsilon$, was determined by plotting the ratio of the observed absorbance, $\mathrm{A}$, at a given wavelength to the concentration, $\mathrm{C}$, as a function of the respective wavelengths that encompass the absorbance spectrum in accordance with the Beer-Lambert's law $(\mathrm{A} / \mathrm{bC})$ where $b$ is the solution thickness or light path length. The $\varepsilon$ was taken at the peak of the plot at which $\lambda=$ $\lambda_{\text {max. }}$ All measurements were made at room temperature, $25 \pm 0.20^{\circ} \mathrm{C}$.

\section{Refractive Index Determination}

The refractive indices, $\mathrm{n}$, of bicontinuous microemulsion and $0.10 \mathrm{M} \mathrm{H}_{2} \mathrm{SO}_{4}$ solutions were determined using the digital Abbe Leica Refractometer which was calibrated with a triply distilled deionized water. The obtained values of these solutions were 1.39757 and 1.34323, respectively.

\section{Density Measurement}

The density of the prepared bicontinuous microemulsion was determined using the digital PAAR density meter, model DMA 35. The value obtained was $0.995 \mathrm{~g} / \mathrm{cm}^{3}$.

\section{Quantum Yield Determination}

The quantum yield, $\phi$, of riboflavin was determined in accordance with the literature methodology ${ }^{29}$. Briefly, the solution of reference standard (quinine sulfate) and riboflavin were prepared to have approximately equal absorbance and were excited at the same wavelength $(356 \mathrm{~nm})$. In a review article on quantum yield determination, Demas and Crosby ${ }^{30}$ comment that the quantum yield of quinine sulfate is constant between the excitation wavelengths of 200 and $390 \mathrm{~nm}$. The value of the quantum yield for this reference sample is recommended to be 0.54 and it was used in this work. This way, the fluorescence emission spectrum of riboflavin solution was corrected for the quantum yield determination using the relation given in equation $1^{31}$.

$$
\frac{\phi_{1}}{\phi_{2}}=\frac{\mathrm{n}_{1}^{2} \mathrm{~A}_{1}}{\mathrm{n}_{2}^{2} \mathrm{~A}_{2}}
$$

In this equation the subcripts 1 and 2 refer to the sample (riboflavin and quinine sulfate), respectively, $\mathrm{A}$ and $\mathrm{n}$ are the area of corrected fluorescence band and refractive index, respectively. Using this relationship, the quantum yield of riboflavin is determined as 0.22 . This value is relatively in agreement with the literature values ${ }^{32-34}$.

\section{Fluorescence lifetime determination}

The fluorescence lifetime, $\tau_{\mathrm{o}}$, for riboflavin in bicontinuous microemulsion was determined using the Strickler-Berg relations ${ }^{34}$ given in equation 2:

$$
\frac{1}{\tau_{0}}=2.88 \times 10^{-9} \mathrm{n}^{2} \overline{v_{f}^{3}} \phi^{-1} \int \varepsilon d \ln v
$$

In this equation $\mathrm{n}, \overline{v_{f}^{3}}$ and $v$ are the solvent (bicontinuous microemulsion) refractive index, the fluorescence wave number, taken at the center of gravity of the fluorescence spectrum, and the integrated wave number of the absorption band, respectively. Riboflavin exhibits an uncomplicated single absorption band whose maximum centered at $415 \mathrm{~nm}$. The integration in equation 2 was therefore done analytically by measuring the area enclosed under the absorbance envelope using the relation of full width at half maximum (FWHM) technique. In this case equation 2 is reduced to a much simpler relation given in equation 3 .

$$
\frac{1}{\tau_{0}}=2.88 \times 10^{-9} \mathrm{n}^{2} \overline{v_{f}^{3}} \phi^{-1} \varepsilon \ln \left(\frac{v_{2}}{v_{1}}\right)
$$

Subscripts 1 and 2 in this equation denote the wavenumbers corresponding to the high and low ends of the wavenumbers of the spectral band and $\varepsilon$ is the molar absorptivity of riboflavin. The determined fluorescence lifetime of riboflavin is $2.68 \mathrm{~ns}$.

\section{Electrochemical measurements}

All the electrochemical measurements were made in a 1-compartment electrochemical cell using a threeelectrode system comprising of the working electrode (1.0 mm diameter) glassy Carbon Electrode (GCE) obtained from cypress Systems, a wound platinum wire as the counter electrode and a commercial calomel electrode as the reference electrode, which was obtained from Fisher Scientific. A computercontrolled electrochemical analyzer system, supplied by cypress Systems, Model CS-10190 was used to obtain the requisite voltammograms. The polisher, Metaserve $₫ 200$, a brown polishing cloth, to resurface the working electrode surface and 0.05 particle size of $\gamma$-aluminum powder, used in conjunction with the polishing cloth were obtained from Buehler Ltd. The water used in all experiments was triply distilled and deionized through the reagent grade water system supplied by Photronix.

\section{Results and Discussion}

We show in Fig. 3(a) the absorbance spectrum of riboflavin. It can be seen that this compound exhibits an uncomplicated, single absorption spectrum from the wavelength of 350 to $500 \mathrm{~nm}$ and the $\lambda_{\max }$ is observed at $415 \mathrm{~nm}$. Fig. 3(b) is its molar absorption spectrum which was described earlier. At this $\lambda_{\max }$ the $\varepsilon$ observed is $1.45 \times 10^{4} \mathrm{M}^{-1} \mathrm{~cm}^{-1}$. This value is in good agreement of literature value ${ }^{34,36,37}$. 


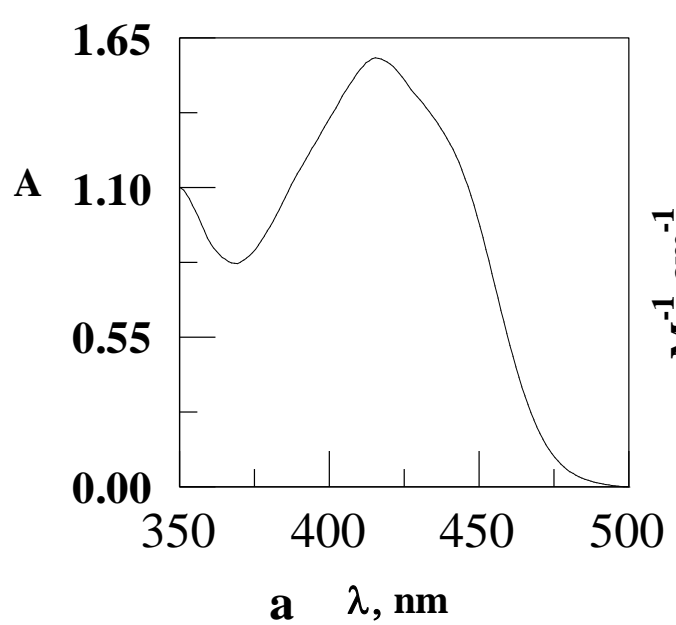

Figure 3(a). Absorbance spectrum of Riboflavin,

The value of the absorption $\lambda_{\max }$ and $\varepsilon$ thus obtained were used in the determination of the fluorescence lifetime of riboflavin given in equation 3.

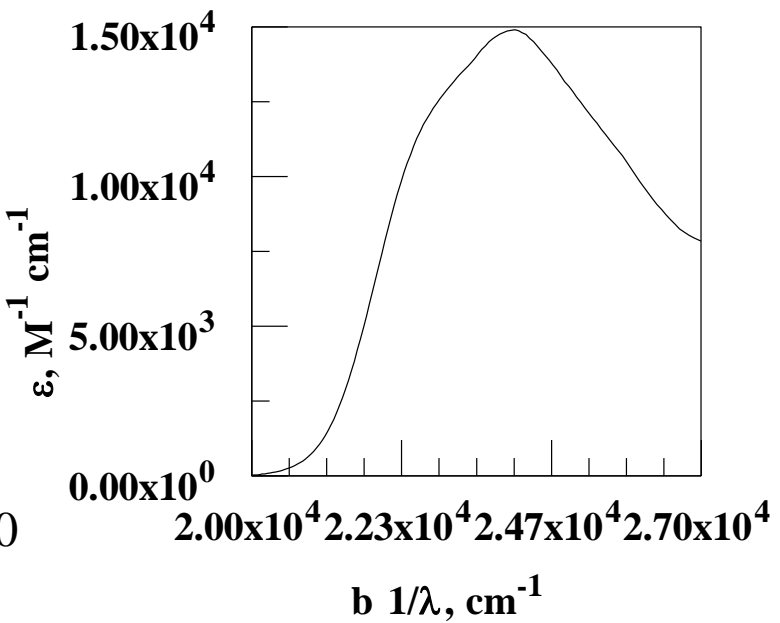

Fig. 3(b). Molar Absorptivity of Riboflavin as a Function of Wavelength

The fluorescence spectra of riboflavin without and with different concentrations of nicotine, the quencher, is shown in Fig. 4.

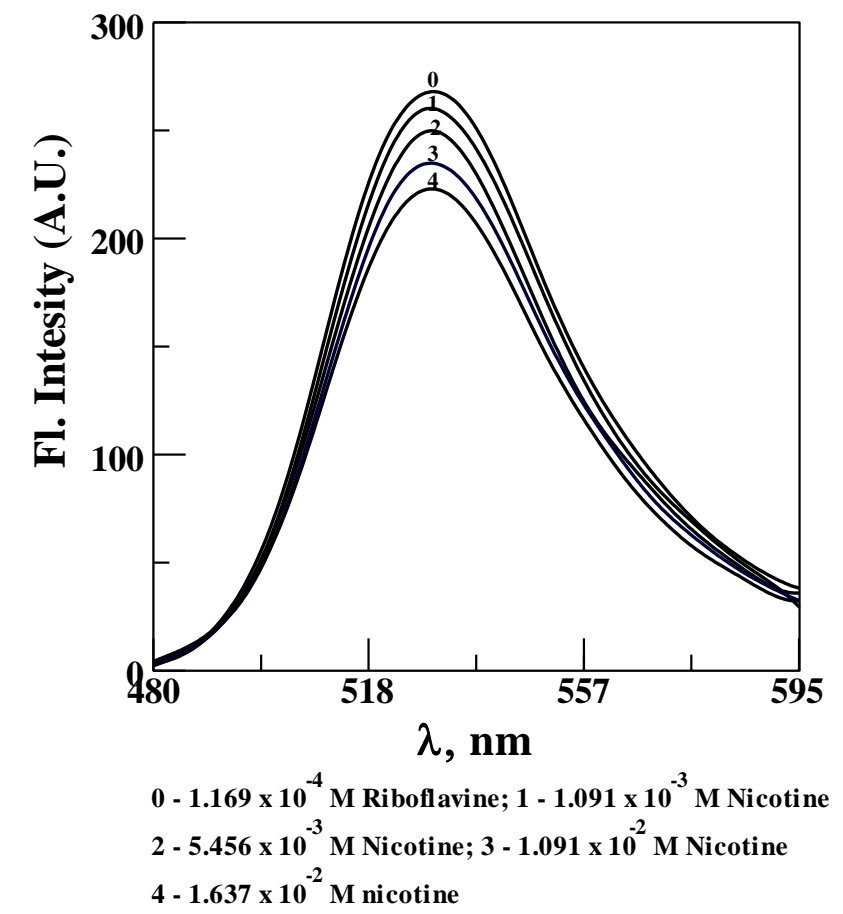

Figure 4. Fluorescence spectra of riboflavin without and with quencher

As can be noticed, the fluorescence of riboflavin is seen to decrease with an increase in the concentration of the quencher as it should be. Analysis of the quenching data was carried out in accordance with the Stern-Volmer relation as given in equation 4.

$\frac{\mathrm{I}^{0}}{\mathrm{I}}=1+\mathrm{K}_{\mathrm{SV}}[$ Nicotine $]=1+\mathrm{k}_{\mathrm{q}} \tau_{0}[$ Nicotine $] \quad 4$
In this equation, $\mathrm{I}^{0}$ and $\mathrm{I}$ are the observed fluorescence intensity in the absence and in the presence of the quencher, respectively. The values of $\mathrm{I}^{0}$ and I were taken at the emission band maximum as is usually the standard practice. $\mathrm{K}_{\mathrm{SV}}$ is the Stern-Volmer quenching constant, $\mathrm{k}_{\mathrm{q}}$ and $\tau_{0}$ are the quenching rate constant and the natural fluorescence lifetime of riboflavin, respectively. The plot of the observed data, per equation 4 is shown in Fig. 5. 


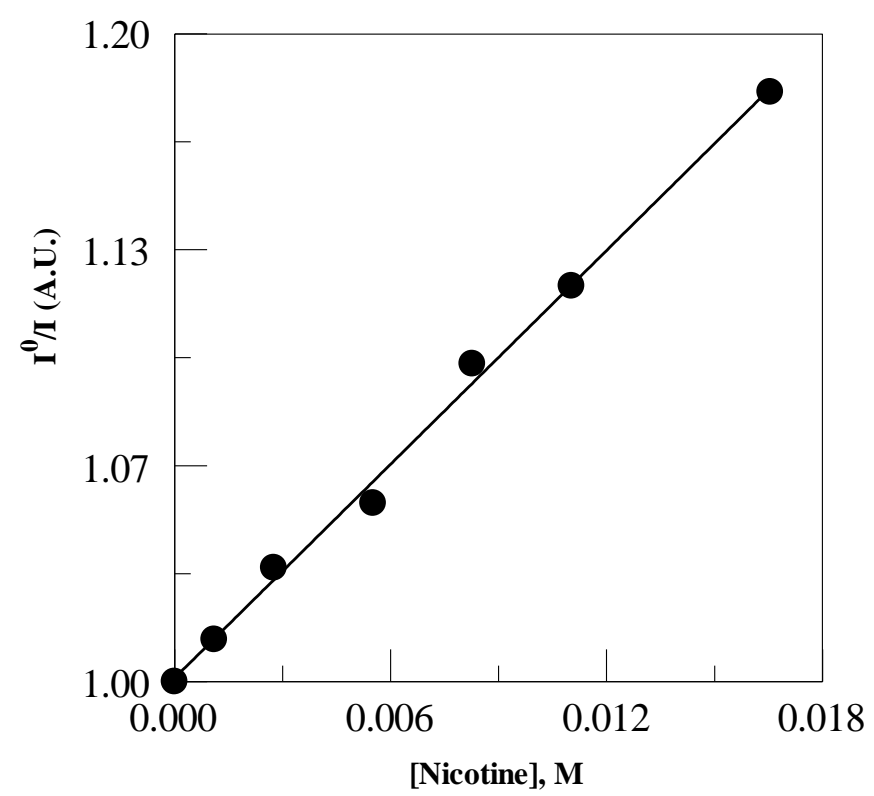

Figure 5. The S-V plot of Riboflavin-Nicotine complex

As can be seen, the plot obeyed the Stern-Volmer equation with a correlation coefficient of better than 0.99 with a slope of $11.12 / \mathrm{M}$, corresponding to the $\mathrm{K}_{\mathrm{SV}}$ as per equation 4 . This value was used with the value of $\tau_{0}$ discussed above, to obtain a quenching rate constant, $\mathrm{k}_{\mathrm{q}}$ of $4.115 \times 10^{9} / \mathrm{M}-\mathrm{s}$. The obtained $\mathrm{k}_{\mathrm{q}}$ was compared with the theoretical bimolecular diffusioncontrolled reaction rate constant, $\mathrm{k}_{\mathrm{d}}$, using the Yguerabide equation given in equation $5^{38-40}$ which is a modification of the Smoluchowski equation which is $4 \pi \mathrm{N}^{\prime} \mathrm{DR}$.

$k_{\mathrm{d}}=4 \pi \mathrm{N}^{\prime} \mathrm{DR}\left[1+\frac{\mathrm{R}}{\left(2 \mathrm{D} \tau_{o}\right)^{1 / 2}}\right]$

$\mathrm{D}$ and $\mathrm{R}$ in this equation refer to diffusion coefficient and radius, respectively and $N^{\prime}$ is the Avogadro number in molecules/mole. However, the Ds in this equation were calculated based on the phase at which the reactants were solubilized. $D_{\text {Riboflavin was }}$ calculated using the density of oil phase, tetradecane, whose density is $0.762 \mathrm{~g} / \mathrm{cm}^{3}$ and the calculated viscosity is $2.07 \mathrm{cP}$ while $\mathrm{D}_{\text {Nicotine }}$ was calculated using the density of water is approximated to 1.0 $\mathrm{g} / \mathrm{cm}^{3}$ and the calculated viscosity is $0.89 \mathrm{cP}$, that is D $=\mathrm{D}^{\mathrm{O}}$ Riboflavin $+\mathrm{D}^{\mathrm{W}}$ Nicotine. This is consistent with what Osaki and his co-workers ${ }^{41}$ suggested. The D thus obtained is $7.97 \times 10^{-6} \mathrm{~cm}^{2} / \mathrm{s}$. The value of the radii of riboflavin and nicotine were calculated using the crystallographic method as given by Smoluchowski ( $\mathrm{R}=\left(\frac{3 \mathrm{M}}{4 \pi \mathrm{N} \rho}\right)^{1 / 3}$ ), where $\mathrm{M}$ and $\rho$ refer to the molar mass and density of the respective compounds. Nicotine's density was taken from ref. $42\left(1.01 \mathrm{~g} / \mathrm{cm}^{3}\right)$ and that of riboflavin is $1.65 \mathrm{~g} / \mathrm{cm}^{3}{ }^{43}$. The diffusion coefficient of the respective molecules was calculated using the Stokes-Einstein equation given in equation 6 .

$$
\mathrm{D}=\frac{\mathrm{kT}}{6 \pi \eta \mathrm{R}}
$$

In this equation $\mathrm{k}$ is the Boltzmann constant, $\eta$ is the viscosity of the Oil and Water media as given above. Using these relations $\mathrm{k}_{\mathrm{d}}$ was determined as $7.36 \times 10 \%$, which is very close to the theoretical diffusion-controlled bimolecular reaction rate constant. The order of magnitude of $\mathrm{k}_{\mathrm{q}}$ and $\mathrm{k}_{\mathrm{d}}$ are equal which signifies that the reaction is indeed diffusion controlled.

\section{Electron transfer rate constant}

According to Sutin ${ }^{44}, \mathrm{k}_{\mathrm{q}}$ and $\mathrm{k}_{\mathrm{d}}$ are related to the electron transfer rate constant $\mathrm{k}_{\mathrm{ET}}$ by the equation: $\mathrm{k}_{\mathrm{q}}=\frac{\mathrm{k}_{\mathrm{ET}} \mathrm{k}_{\mathrm{d}}}{\mathrm{k}_{\mathrm{ET}}+\mathrm{k}_{-\mathrm{d}}}$

$\mathrm{k}_{-\mathrm{d}}$ in this equation is the first order diffusioncontrolled dissociation rate constant of the precursor complex shown in the following scheme:

$$
\mathrm{D}+\mathrm{hv} \rightarrow \mathrm{D}^{*} \quad ; \quad \mathrm{D}^{*}+\mathrm{A} \underset{\mathrm{k}_{-\mathrm{d}}}{\stackrel{\mathrm{k}_{\mathrm{d}}}{\rightleftarrows}}
$$

Rearrangement of equation 7 in accordance with the Sutin formalism, loc. Cit., gives the following relation:

$$
\frac{1}{\mathrm{k}_{\mathrm{q}}}=\frac{1}{\mathrm{k}_{\mathrm{d}}}+\frac{1}{\mathrm{~K}(\mathrm{R}) \mathrm{k}_{\mathrm{ET}}}
$$

$\mathrm{k}_{\mathrm{ET}}$ is the first- order electron transfer rate constant and $\mathrm{K}(\mathrm{R})$ equals to $\mathrm{k}_{\mathrm{d}} / \mathrm{k}_{-\mathrm{d}}$ is the distant dependent association or equilibrium constant of the precursor complex that is obtained using the Fuoss and Debye model 45,46 given in equation 9 for uncharged reactants as is the case in this work: 


$$
\mathrm{K}(\mathrm{R})=\frac{4 \pi \mathrm{NR}^{3}}{3000}
$$

The value of $\mathrm{K}(\mathrm{R})$ thus calculated is $1.615 \mathrm{M}^{-1}$, where the $\mathrm{R}$-value is approximated by the sum of the

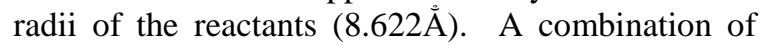
equations 7-9 was used to obtain $\mathrm{k}_{\mathrm{ET}}$ of $5.89 \times 10^{9} \mathrm{~s}^{-1}$. This value is consistent with what is expected for long range photo induced electron transfer reactions within an encounter radius $\geq 7 \stackrel{\Delta}{4} 470$. The activationcontrolled rate constant, $\mathrm{k}_{\mathrm{a}}$, of $9.52 \times 10^{9} \mathrm{~s}^{-1}$ for the reaction under study was determined using the Sutin equation ${ }^{44}: \mathrm{k}_{\mathrm{a}}=\mathrm{K}(\mathrm{R}) \mathrm{k}_{\mathrm{ET}}$. A comparison of the magnitude of $\mathrm{k}_{\mathrm{a}}$ with $\mathrm{k}_{\mathrm{q}}, \mathrm{k}_{\mathrm{d}}$ and $\mathrm{k}_{\mathrm{ET}}$ reveals some relevant facts in this work: $\mathrm{k}_{\mathrm{q}} \approx \mathrm{k}_{\mathrm{a}}$ and $\mathrm{k}_{\mathrm{ET}}<\mathrm{k}_{\mathrm{d}}$. This observation implies an activation-controlled reaction process and the observed $\mathrm{k}_{\mathrm{ET}}$ is within a value in a solvent separated radical ion pair reaction and the quenching efficiency of riboflavin by nicotine in bicontinuous microemulsion, $\left(\gamma=\frac{\mathrm{k}_{\mathrm{q}}}{\mathrm{k}_{\mathrm{d}}}\right)^{51}$ is $56 \%$.

This implies that only $56 \%$ of all the collisions of riboflavin with nicotine result in quenching of riboflavin fluorescence.

\section{Solvent reorganization energy}

In order to fully understand the observed electron transfer mechanism, the solvents' involvement is considered. For the calculation of the solvent reorganization energy, $\lambda_{\mathrm{s}}$, we have used the Marcus dielectric continuum formula ${ }^{52}$ given in equation 10 :

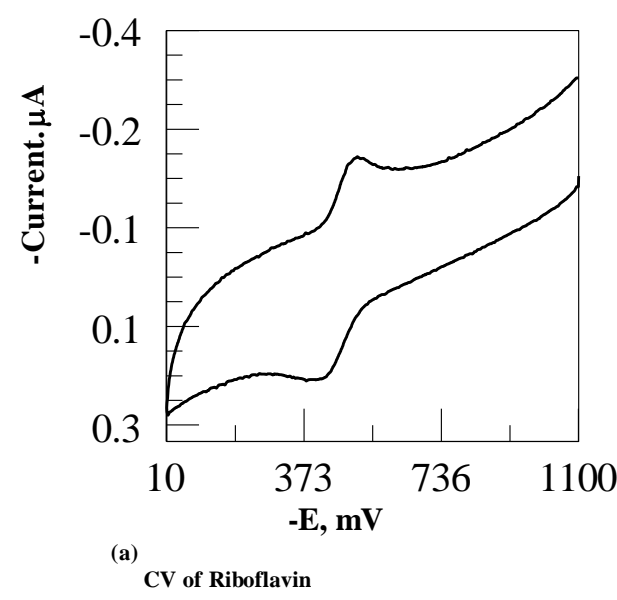

$$
\lambda_{\mathrm{s}}=\frac{\mathrm{e}^{2}}{4 \pi \varepsilon_{0}}\left(\frac{1}{2 \mathrm{r}_{\mathrm{A}}}+\frac{1}{2 \mathrm{r}_{\mathrm{D}}}-\frac{1}{\mathrm{R}}\right)\left(\frac{1}{\varepsilon_{\mathrm{op}}^{2}}-\frac{1}{\varepsilon_{\mathrm{s}}}\right)
$$

In this equation $\varepsilon_{\mathrm{op}}^{2}=\mathrm{n}$ and $\varepsilon_{\mathrm{s}}$ are the solution refractive index and the corresponding dielectric constant, respectively. The dielectric constant of the prepared bicontinuous microemulsion was experimentally determined as discussed in the experimental section. The dielectric constant of this medium was taken from ref 53. e is the electronic charge and $\varepsilon_{o}$ is the permittivity of vacuum. The use of this equation gave a value of $1.09 \mathrm{eV}$ for the solvent reorganization energy of riboflavin-nicotine reaction in the bicontinuous microemulsion. This value is within the range of those values that implicate a solvent separated reaction process ${ }^{50}$.

\section{Free energy change}

In addition to the solvent reorganization energy, the free energy change, $\Delta \mathrm{G}^{\mathrm{o}}$, for the electron transfer reaction in this medium is also determined using the Rehm-Weller relation ${ }^{54}$ given in equation 11 :

$$
\Delta \mathrm{G}^{\mathrm{o}}=\mathrm{E}_{\frac{1}{2}}^{\mathrm{ox}}-\mathrm{E}_{\frac{1}{2}}^{\mathrm{Red}}-\mathrm{E}_{0-0}-\frac{\mathrm{e}^{2}}{4 \pi \pi_{\mathrm{o}} \varepsilon_{\mathrm{s}} \mathrm{R}_{\mathrm{q}}}
$$

In the above equation $\mathrm{E}_{\frac{1}{2}}^{\mathrm{ox}}$ and $\mathrm{E}_{\frac{1}{2}}^{\mathrm{Red}}$ are the halfwave potentials of the oxidant and reductant, respectively. We show in Fig. 6 (a) \& (b) the voltammograms of $1.0 \mathrm{mM}$ Nicotine and $0.92 \mathrm{mM}$ of riboflavin in bicontinuous microemulsion used in the calculation of $\mathrm{E}^{1} / 2$.

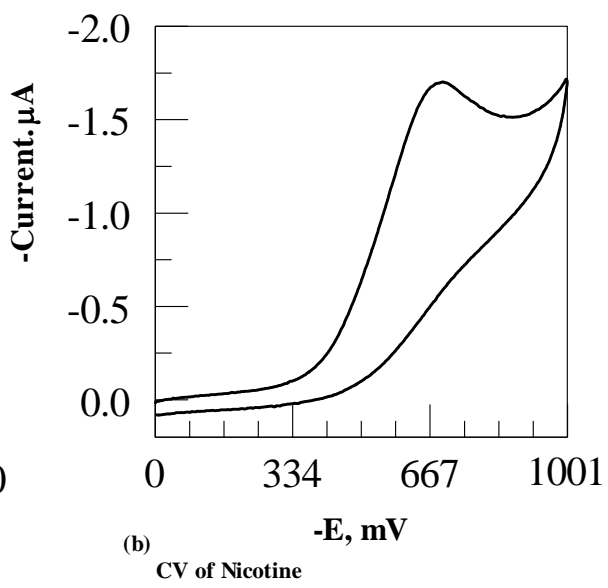

Figure 6(a) \& (b). The Voltammograms of Riboflavin and Nicotine.

The data from these voltammograms were used to determine the $\mathrm{E}_{1 / 2}$ of the respective molecules according to equation $12^{55}$ :

$$
\mathrm{E}=\mathrm{E}_{1 / 2}+\frac{\mathrm{RT}}{\mathrm{nF}} \ln \left(\frac{\mathrm{i}}{\mathrm{i}_{\mathrm{d}}-\mathrm{i}}\right)=\mathrm{E}_{1 / 2}+\frac{0.059}{\mathrm{n}} \log \left(\frac{\mathrm{i}}{\mathrm{i}_{\mathrm{d}}-\mathrm{i}}\right)
$$

A plot of the observed potential, E, as a function of the logarithmic the ratio of the observed current versus the diffusion current, $i_{d}$, that is,

(E vs $\log \left(\left(i / i_{d}-i\right)\right)$ usually gives a straight line (see Figures 7(a) \& (b) whose intercept is taken as halfwave potential, $\mathrm{E}_{1 / 2}$. 

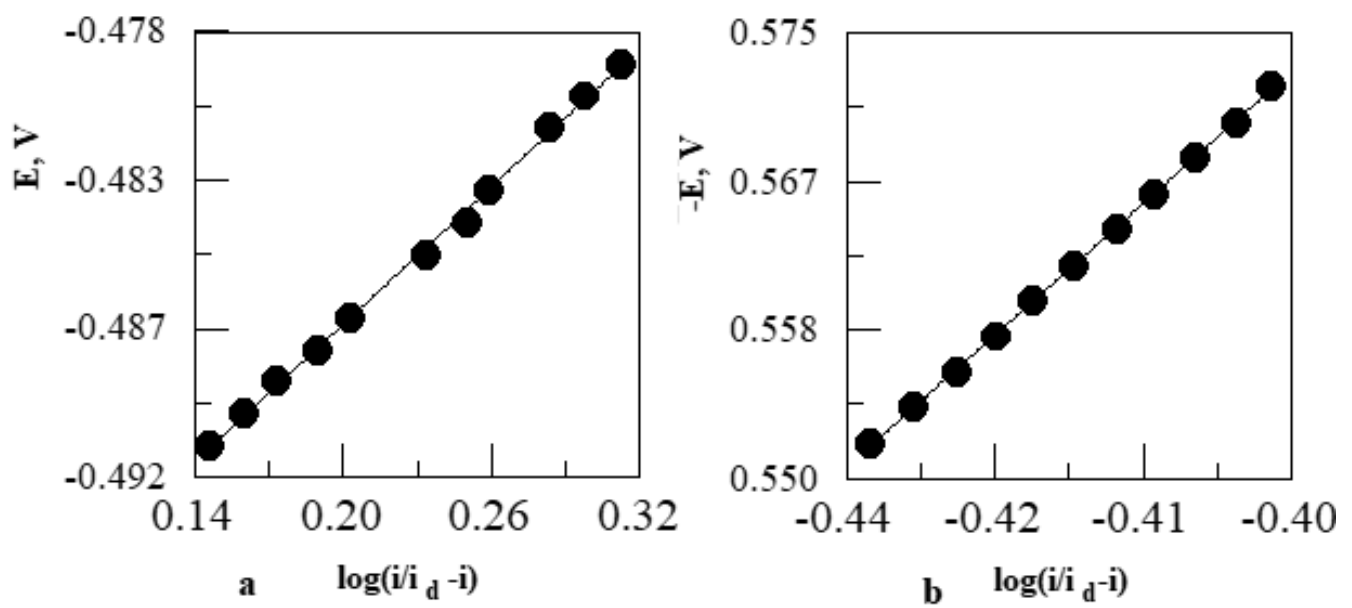

Figures 7(a) \& (b). The plot of the observed potential (E) versus the lag of the current ratio of Riboflavin and Nicotine

This is the usual electrochemical technique used for the determination of $\mathrm{E}_{1 / 2}$ for a quasi-reversible and an irreversible electrochemical reaction, as the voltammograms of riboflavin and nicotine show (Fig. 6(a) and (b). Using this relation, the $\mathrm{E}^{1} / 2$ observed for nicotine and riboflavin are $-0.78 \mathrm{~V}$ and -0.50 , respectively. E o-o is the zero-zero excitation energy of riboflavin taken at the maximum of the excitation spectrum $(401 \mathrm{~nm})$. The $\mathrm{Rq}$ in equation 11 , as modified by Fayer and his co-workers ${ }^{56}$ equation 11 , is given in equation 13 was used to calculate $\mathrm{Rq}$

$\mathrm{R}_{\mathrm{q}}=\mathrm{R}+\frac{\mathrm{L}}{2} \ln \left(\frac{\gamma^{2} \mathrm{~W}_{\infty} \mathrm{L}^{2}}{4 \mathrm{D}}\right)$

$\mathrm{R}_{\mathrm{q}}$ in this equation is the effective electron transfer distance between the reactants including the monolayer of the amphiphilic molecule $(\mathrm{CTAB})$ that is sandwiched between the water and the oil phases.
$\mathrm{R}=\mathrm{r}_{\text {riboflavin }}+\mathrm{r}_{\text {nicotine }}, \mathrm{L}$ is an electron tunneling length, $\gamma=\operatorname{expC}$ where $\mathrm{C}$ is the Euler constant $(0.577) . \mathrm{W}_{\infty}=$ $1.8 \times 10^{10} / \mathrm{s}$ and $\mathrm{D}$ is the sum of diffusion coefficients of the molecules using the density of the bicontinuous microemulsion $\left(9.582 \times 10^{-7} \mathrm{~cm}^{2} / \mathrm{s}\right)$. Putting these values into equation 13 yields the effective quenching radius, $R_{\mathrm{q}}$, as $9.10 \times 10^{-8} \mathrm{~cm}$ and the resulting $\Delta \mathrm{G}^{\circ}$ in equation 11 is calculated as $-2.9 \mathrm{eV}$. Having determined the $\Delta G^{o}$ and $\lambda_{s}$, the free energy of activation as per Marcus continuum theory, loc. Cit., is given in equation 14 .

$\mathrm{G}^{*}=\frac{\left(\lambda+\mathrm{G}^{\mathrm{o}}\right)^{2}}{4 \mathrm{D}}$

The value thus obtained is $0.75 \mathrm{eV}$. The observed parameters are consistent with outer sphere, electron transfer process. Table 2 shows the relevant parameters determined in this work.

Table 2. Observed/Calculated Parameters for Riboflavin/Nicotine Complex in Bicontinuou Microemulsion.

\begin{tabular}{|c|c|}
\hline Parameter & Value \\
\hline 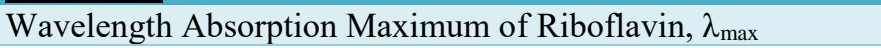 & $\overline{415 \mathrm{~nm}}$ \\
\hline Molar Absorptivity of Riboflavin, $\varepsilon$ & $1.45 \times 10^{4} \mathrm{M}^{-1} \mathrm{~cm}^{-1}$ \\
\hline Fluorescence emission wavelength maximum of Riboflavin & $529 \mathrm{~nm}$ \\
\hline Fluorescence excitation wavelength of Riboflavin & $401 \mathrm{~nm}$ \\
\hline Refractive index of Riboflavin in bicontinuous microemulsion & 1.39757 \\
\hline Density of CTAB, $\rho$ & $0.995 \mathrm{~g} / \mathrm{cm}^{3}$ \\
\hline Quantum yield of Riboflavin & 0.22 \\
\hline Fluorescence life time of Riboflavin & 2. $68 \mathrm{~ns}$ \\
\hline Half-wave potential of Riboflavin, $E_{1 / 2}$ & $-0.58 \mathrm{~V}$ \\
\hline Half-wave potential of Nicotine, $E_{1 / 2}$ & $-0.78 \mathrm{~V}$ \\
\hline Quenching constant, $\mathrm{K}_{\mathrm{SV}}$ & $11.12 \mathrm{M}^{-1}$ \\
\hline Quenching rate constant, $\mathrm{K}_{\mathrm{q}}$ & $4.115 \times 10^{9} \mathrm{M}^{-1} \mathrm{~s}^{-1}$ \\
\hline Theoretical bi-molecular diffusion-controlled rate constant, $\mathrm{k}_{\mathrm{d}}$ & $7.36 \times 10^{9} \mathrm{~s}^{-1}$ \\
\hline Precursor equilibrium Constant, $\mathrm{K}$ & $1.615 \mathrm{M}^{-1}$ \\
\hline Electron transfer rate constant $\mathrm{k}_{\mathrm{ET}}$ & $5.89 \times 10^{9} \mathrm{~s}^{-1}$ \\
\hline Free energy of reaction, $\Delta \mathrm{G}^{\mathrm{o}}$ & $-2.9 \mathrm{eV}$ \\
\hline Free energy of activation, $\Delta \mathrm{G}^{*}$ & $0.75 \mathrm{eV}$ \\
\hline Solvent reorganization energy, $\lambda_{\mathrm{s}}$ & $1.09 \mathrm{eV}$ \\
\hline Effective electron transfer distance, $\mathrm{R}_{\mathrm{q}}$ & $9.10 \times 10^{-8} \mathrm{~cm}$ \\
\hline Efficiency of quenching, $\gamma$ & $51 \%$ \\
\hline
\end{tabular}


Using these values, we propose in the scheme below, a plausible quenching mechanism of riboflavin

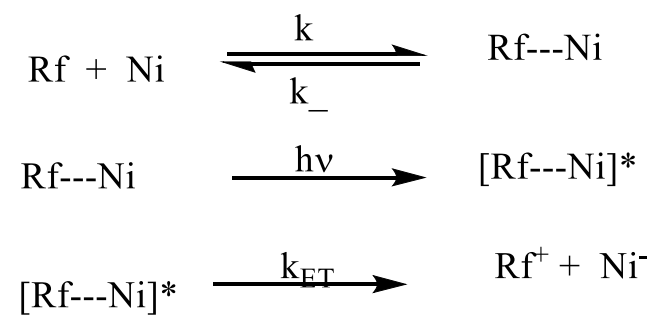

\section{Conclusion}

It has been shown in this work that the interaction of nicotine with riboflavin in the prepared bicontinuous microemulsion leads to the quenching of riboflavin fluorescence. The observed and calculated data are reasonably in agreement with an activated and diffusion-controlled electron transfer mechanism. The free energy of reaction, $\Delta \mathrm{G}^{\mathrm{o}}$, the free energy of activation, $\Delta \mathrm{G}^{*}$, and the activation energy rate constant, $\mathrm{k}_{\mathrm{a}}$, and other pertinent parameters in this ET reaction were determined and tabulated in Table 2.

\section{Acknowledgement}

The author is very grateful to Chemistry Department and the Morgan State University for their support of this work.

\section{References}

1- J.M. Grippa, et al., J. Agricultural and Food Chemistry 2014, 62, 1153-1158.

2- B. Koziol, M. Markowicz, J. Kruk, B. Plytycz, Photochem Photobiol 2006. 82, 570-573.

3- S. Hustad, M.C. McKinley, H. McNulty, et al., Clinical Chemistry, 2002, 48(9), 1571-1577.

4- MedlnePlus http:www.nih.gov/medlineplus/druginfo/natural 1957.html, 2012.

5- $\quad$ http://umm.edu/health/medical/altmed/supplem ent/vitamin-b2-riboflavin.

6- W.S.A. Innis, D.B. McCormick, A.L. Merrill, Biochemical Medicine 1985, 34, 151-165.

7- H.J. Powers, Am. J. Clin. Nutr. 2003. 77, 1352-1360.

8- V. Massey, Biochemical Society Transactions 2000, 28, part 4, 283-296.

9- M.Y. Jung, Y.S. Oh, D.K. Kim, H.J. Kim, D.B. Min, J. Agric. Food Chem. 2007, 55, 170-174.

10- T.J. Dickkerson, N. Yamamoto, K.D. Janda, Bioorganic \& Medicinal Chemistry 2004, 12, 4981-4987.

11- M.B. Babior, C. Takeuchi, J. Ruedi, A. Gutierrez, P. Wentworth Jr, PNAS 2003 , 100(6), 3031-3034.

12- A.D. Wentworth, L.H. Jones, P. Wentworth, K.D. Janda, R.A. Lerner, PNAS 2000, 97(20), 10930-10935.
(Rf) by nicotine $(\mathrm{Ni})$.

13- J. Wang, J.M. Kim, D.M. Donovan, K.C. Becker, M.D. Li, Mitochondrion 2009, 9, 186-195.

14- W.A. Pryor, N.C. Arbour, B. Upham, D.F. Church, Free Radical Biology\& Medicine 1992, 12, 365-372.

15- Nicotine Chemistry, Chemistry Daily.com 2005.

16- World of Molecules http://www.worldof molecules.com/drugs/nicotine.htm Nicotine 2014.

17- M.L. Solt, Plant Physiology 1957, 32, 480-484.

18- M.L. Solt, Ibid 1957, 32, 484-490.

19- R.C. Jiloha, Indian J. Psychiatry 2010, 52(4), 301-307.

20- R. Schartz-Bloom, G.G. de Nunez, www.pbs.org/wgbh/nova/cigarette/nicotine_nfp htm.

21- N.L. Benowitz, J. Hukkanen, P. Jacob III, Nicotine Psychopharmacology J. E. Henningfield, E. Calvento S. Pogun (Eds) 2009, 29-60.

22- S. Banerje, G. Bandyopadhyaya, K. Chattopadhyay, B.D. Chattopadhyay, Int. J. Pharmacology 2010, 6(4) 444-455.

23- J.A. Dani, J.I. Daoyun, F.M. Zhou, Neuron 2001, 31, 348-352.

24- D.J.K. Balfour, A.E. Wright, M.E.M. Benwell, C.E. Birrell, Behavioural Brain Research 2000, 113, 73-83.

25- Y. Wang, H.F. Sun, H.F. Wang, Y.F. Liu, Chinese Chemical Letters 2001, 12(5), 449-452.

26- S.K. Mahapatra, S. Das, S. Bhattacharjee, N. Gautam, S. Majumdar, S. Roy, Toxicology Mechanism and Methods 2009, 19(2), 100-108.

27- K. Chattopadhyay, B.D. Chattopadhyay, Indian J. Med. Res. 2008, 127, 571-576.

28- R. Rusakowiez, A.C. Testa, The J. Phys. Chem. 1968, 72(8), 793-796.

29- J.N. Demas, G.A. Crosby, Ibid 1971, 75(8), 991-1024.

30- S.D.M. Islam, A. Penzkofer, P. Hegemann Chemical physics 2003, 291, 97-114.

31- A. Filipowicz, S. Wolowicz, Int. J. Pharmaceutics 2003, 408, 152-156.

32- G. Weber, F.W.J. Teale, Trans Faraday Soc. 1957. 33, 646-655.

33- J. Koziol, Photochemistry and Photobiology 1966, 5, 41-54. 
34- W. Holzer, A. Penzkofer, M. Fuhrmann, P. Hegemann, Ibid 2002, 75(5), 479-487.

35- S.J. Strickler, R.A. Berg, The J. Chem. Physics 1962, 37(4), 814-822.

36- N.M. Al-Shaalan, Oriental J. Chem 2015, 31 (4), 1-7.

37- S. Prahl, http://omlc.org/spectral/PhotochemCad/data/00 4.abs.txt, 2012.

38- J. Youerabide, M.A. Dillon, M. Burton, The J. Chem. Physics 1964, 40(10), 3040-3052.

39- R.M. Mevanki, N.R. Patil, H.D. Patil, R.A. Kusanur, J.S. Kadadevaramath, Indian J. of Pure \& Applied Physics 2011, 49, 748-753.

40- J.S. Kadadevarmath, T.P. Giraddi, G.H. Malimath, G.C. Chikkur, Radiation Measurements 1996, 26(1), 117-121.

41- 41 T. Osakai, H. Hotta, T. Sugihara, K. Nakatani, J. Electroanalytical Chem. 2004, 571, 201-206.

42- http:// wikipedia.org/wiki/Nicotine.

43- Santa Cruz Biotech, http://scbt.com/datasheet205906-riboflavin.htm.

44- N. Sutin, Acc. Chem. Res. 1982, 15, 275-282.

45- R.M. Fuoss, J. A. Chem. Soc. 1958, 80, 5059-5061.
46- P. Debye, Trans Electrochem. 1942, 82(1), 265-272.

47- K. Kikuchi, J. Photochem. Photobiol. A: Chem. 1992. 65, 149-156.

48- K. Kikuchi, et al., Chemical Physics Letters 1991, 180()5), 403-408.

49- J.M. Chen, T.I. Ho, C.Y. Mou, J. Phys. Chem. 1990, 94(7), 2889-2896.

50- I.R. Gould, S. Farid, Acc. Chem. Res. 1996, 29, 522-528.

51- J.R. Lakowicz, Principles of Fluorescence Spectroscopy, Plenum Press 1983, pp. 263.

52- R.A. Marcus, The J. Chem. Physics 1965, 43(2), 679-701.

53- M.O. Iwunze, Physics and Chemistry of Liquids 2005, 43(2), 195-203.

54- D. Rehm, A. Weller, Israel J. Chem. 1970, 8, 259-271.

55- A.J. Bard, L.R. Falkner Electrochemical Methods: Fundamentals and Applications. $2^{\text {nd }}$ Ed., John Wiley and Sons. 2001, pp. 273.

56- V.S. Gladkikh, A.L. Burshtein, H.L. Tevernier, M.D. Fayer, J. Phys. Chem. A 2002, 106, 6982-6990. 